
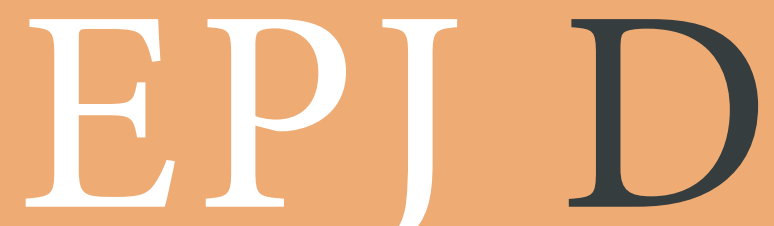

\title{
Atomic, Molecular,
} Optical and Plasma Physics $\quad$ EPJ.ors

Eur. Phys. J. D 62, 57-66 (2011)

DOI: $10.1140 / \mathrm{epjd} / \mathrm{e} 2010-10314-9$

Dynamics of two coupled chaotic systems driven by external signals

H. Mancini and G. Vidal
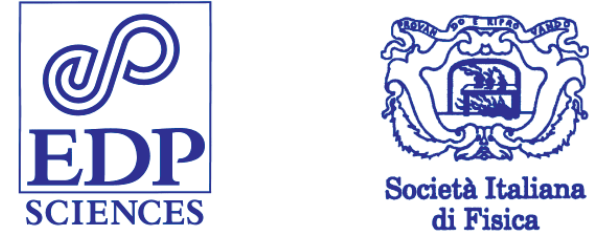

算 Springer

di Fisica 


\title{
Dynamics of two coupled chaotic systems driven by external signals
}

\author{
H. Mancini ${ }^{\mathrm{a}}$ and G. Vidal \\ Instituto de Física, Universidad de Navarra, Irunlarrea s/n, 31080 Pamplona, Spain
}

Received 24 May 2010 / Received in final form 14 September 2010

Published online 10 November 2010 - (C) EDP Sciences, Società Italiana di Fisica, Springer-Verlag 2010

\begin{abstract}
Setting-up a controlled or synchronized state in a space-time chaotic structure targeting an unstable periodic orbit is a key feature of many problems in high dimensional physical, electronics, biological and ecological systems (among others). Formerly, we have shown numerically and experimentally that phase synchronization [M.G. Rosenblum, A.S. Pikovsky, J. Kurths, Phys. Rev. Lett. 78, 4193 (1997)] can be achieved in time dependent hydrodynamic flows [D. Maza, A. Vallone, H.L. Mancini, S. Boccaletti, Phys. Rev. Lett. 85, 5567 (2000)]. In that case the flow was generated in a small container with inhomogeneous heating in order to have a single roll structure produced by a Bénard-Marangoni instability [E.L. Koshmieder, Bénard Cells and Taylor Vortices (Cambridge University Press, 1993)]. Phase synchronization was achieved by a small amplitude signal injected at a subharmonic frequency obtained from the measured Fourier temperature spectrum. In this work, we analyze numerically the effects of driving two previously synchronized chaotic oscillators by an external signal. The numerical system represents a convective experiment in a small container with square symmetry, where boundary layer instabilities are coupled by a common flow. This work is an attempt to control this situation and overcome some difficulties to select useful frequency values for the driving force, analyzing the influence of different harmonic injection signals on the synchronization in a system composed by two identical chaotic Takens-Bogdanov equations (TBA and TBB) bidirectionally coupled.
\end{abstract}

\section{Introduction}

Time dependent patterns in far from equilibrium systems are very common in many fields of science like physics, chemistry, biology and engineering [4]. Convection in fluids, plasmas, crystallization processes, wide aperture semiconductor lasers, or chemical reaction-diffusion systems are only a few of technologically important examples where control and synchronization of space-time chaotics flows could be important [5].

Stabilization on these kind of flows could be necessary in both, uniform or patterned unstable states when deposition of thin or thick films on a substrate are from a liquid phase. It is usually important to control the liquid phase before deposition to avoid defects or cracks in the film or to reproduce a desired matrix of flows to improve the process. In other applications like mixing in microfluidics, the useful state could be the chaotic one in order to improve the mixing results.

To optimize both kind of processes it is necessary to control them in order to select one of the suitable states, even if they are naturally unstable.

There are experimental problems to control or synchronize a chaotic time dependent spatial state in an ex-

\footnotetext{
a e-mail: hmancini@fisica.unav.es
}

periment, and among them, probably the more relevant are to choose the sampling frequency and the minimum number of spatial points to feedback the control signals. Most of the theoretical methods assume that all locations in an experiment are accessible, something difficult to accomplish in real experiments.

The magnitude of the problem of determining the minimum number of controllers can be understood considering that in some numerical simulations [6] synchronization in a time dependent chaotic state between two identical Hele-Shaw cells heated from below, was impossible with less than 700 points of interconnection, clearly a situations almost impossible to be get experimentally in thermal convection.

Theory and experiments have shown that space-time control acting on relatively few points is possible if the space-time dynamics equations of the system are well known [7]. In that case, many efforts have been done during the last ten years to develop a general control algorithm for space-time chaotic systems, principally considering modeling equations [8,9], coupled map lattices [10] or synchronization using a complex networks approach [11].

Control of real physical systems described by analytical solutions derived from first principles (like NavierStokes equation), usually present problems to fit with the 
experiments, because time-dependent regimes normally appear at high supercritical control parameter values, where a perturbative method can not be applied and many approximations must be done. Normally we work with approximated models and to control a previously targeted unstable chaotic state becomes a hard task. But experimentalists can observe real data from outputs, symmetries in patterns or instability regions and long signal sequences in previously choose positions can be obtained. This is the common approach used to reconstruct the phase space from an experiment, and selecting sampling positions and frequencies.

A numerical model to simulate in advance the experiment is always a very useful tool for designing, even if it is approximate.

In this work, we numerically analyze the problem of driving a synchronized state between two identical chaotic oscillators. A normal situation in thermal convection is to have quasiperiodic dynamics going to space-time chaos in pre-turbulent states when the control parameter is increased sufficiently. Under this conditions, the Fourier spectrum for a variable (like temperature) displays overimposed to the wide band frequencies (typical for a preturbulent state) an enhancement around the band of frequencies that previously have been dominant.

We reconstruct this situation coupling two hyperchaotic oscillators modeled by a system of equations that previously was used to represent time-dependent convection in a small aspect ratio container (SAR) with square symmetry $D_{4}[12-14]$.

The problem of analyzing the driving of two coupled oscillators was recently reconsidered by Anishenko et al. [15] who looks for the effects on phase synchronization of two coupled Van der Pol oscillators when they are forced by an external harmonic signal.

In the same direction, we use here a numerical approach to study a dynamical system representing our experiment driven by different external harmonic signals with the following questions: is there something similar to a "resonance" in (hyper)chaotic systems? How can we detect this resonance in a wide band experimental spectrum?

To answer these questions, the paper is organized as follows: Section 2 presents some details about the dynamics of the coupled system, followed by other details about the synchronization and the problems that we had to obtain useful data from an experimental Fourier spectrum. Section 3 presents details about the results obtained for different driving forces related to the signals used. In Section 4 we include the effect of noise (correlated and noncorrelated). Conclusions close the paper in Section 5 .

\section{The oscillating system}

Two identical oscillators A and B have been coupled to obtain the chaotic synchronized dynamical system that we will drive. Each one of them is a four dimensional Takens-Bogdanov's ODE system. The symmetry properties of the dynamical equations are described by the symmetry group $D_{4}$ composed by reflection $\tau$ and rotation $\rho$

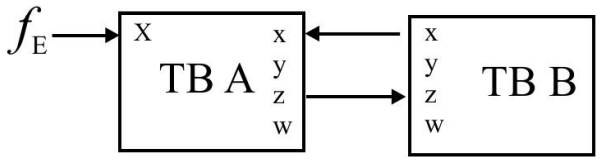

Fig. 1. Interconnection scheme. The harmonic signal $f_{E}$ is always injected to the variable $x$ of oscillator A.

as is described in equation (1):

$$
\begin{array}{ll}
\tau: & (x, y, z, w) \rightarrow(z, w, x, y) \\
\rho: & (x, y, z, w) \rightarrow(-x,-y, z, w) .
\end{array}
$$

These symmetries play an important role in the way that we have to connect A and B, as it is shown in Figure 1.

As it was shown in [12] the patterns in the experiment follows the same symmetry breaking bifurcations sequence described by the Takens-Bogdanov equations [16]. This equation has a Codimension 2 point at the origin and increasing the control parameter (associated with temperature gradient) the pattern becomes time-dependent after a pitchfork bifurcation preserving a part of the original symmetry (a diagonal of the square). Time dependent states are firstly chaotic oscillations in one of the two symmetrical branches (one of these diagonals), and then by an heteroclinic connection, recovers all the space for the variables hidden by the pitchfork involving also oscillations in the other diagonal. Details about the dynamics and the experiments related can be found in cited references.

Looking more in depth this set of equations we observe for the heteroclinic connection parameters that it could be considered composed by two bi-dimensional non-linearly coupled oscillators. This detail is important for choosing a coupling scheme that preserves the original symmetry.

The ODE system for this oscillator is described in equation (2),

$$
\begin{aligned}
& \dot{x}=y \\
& \dot{y}=\mu x+x\left(a\left(x^{2}+z^{2}\right)+b z^{2}\right) \\
& \dot{z}=w \\
& \dot{w}=\mu z+z\left(a\left(x^{2}+z^{2}\right)+b x^{2}\right)
\end{aligned}
$$

where $(x, y, z, w)$ are the variables and $a, b$ and $\mu$, the parameters that have to be adjusted to fit the experiment. In time dependent regimes the system shows a complex chaotic behavior, with more than one positive Lyapunov exponent (or unstable directions) near a fix point (hyperchaos) [17]. Calculation of the Lyapunov exponents, eigenvalues and eigenvectors are necessary to determine the synchronization regions and it has been published previously [18]. An important fact to mention is that this dynamical system (with a riddle basin) is not an attractor, neither by Milnor's definition nor Lyapunov's one $[19,20]$. It is a non-attracting set in some volume of the space, centered on the origin, and works as a repellor in the rest of the space. This fact has implications on dynamics solutions that can really be observed in an experiment. In the experiment, provided that the control parameter is not too high, convection is forced to keep the symmetry by the 
square boundary conditions and to remain in a confined region of variables. Not all the possible mathematical solutions have been observed experimentally. If the control parameter is too high, the preturbulent state is destroyed together with the pattern, appearing waves and bubbles. This set of equations cannot represent this new state.

\section{The coupled system}

Symmetrical coupling of two identical Takens-Bogdanov through one variable $x$ has been presented in reference [18]. Synchronization regime has been analyzed using different coupling schemes (symmetric or asymmetric) and considering the coupling as a direct function of the error between both systems (acting as a feedback loop). Phase synchronization (PS) has been obtained using selected values for parameters that must be fine-tuned to fit the Lyapunov exponent windows [21]. The synchronized regimes obtained in this case are not very stable and depend strongly on the coupling coefficient value.

Here we present a different approach. To obtain a robust synchronization manifold we recover for the coupled system the symmetry of each one oscillator. This is achieved by coupling the feedback on two different variables $(x$ and $z$ ). Internal symmetry of the equations couples variables by pairs ( $x$ and $y$ to $z$ and $w$ ). Introducing the coupling bidirectionally between $\mathrm{A}$ and $\mathrm{B}$ through $x$ and $z$, we are constructing a closed "ring structure" that provides the robustness and complete synchronization (CS) is obtained. This was impossible to obtain in the case of a symmetrical coupling scheme based on only one variable [22]. The equations for the coupled system are now transformed in equation (4), where variables are named by the subscripts (A, B) corresponding to each original system. The coupled system is again hyperchaotic (having more than one positive Lyapunov exponent) and chaos is not completely suppressed by synchronization.

$$
\begin{aligned}
\dot{x}_{A} & =y_{A}+\varepsilon_{x}\left(x_{B}-x_{A}\right) \\
\dot{y}_{A} & =\mu x_{A}+x_{A}\left(a\left(x_{A}^{2}+z_{A}^{2}\right)+b z_{A}^{2}\right) \\
\dot{z}_{A} & =w_{A} \\
\dot{w}_{A} & =\mu z_{A}+z_{A}\left(a\left(x_{A}^{2}+z_{A}^{2}\right)+b x_{A}^{2}\right) \\
\dot{x}_{B} & =y_{B} \\
\dot{y}_{B} & =\mu x_{B}+x_{B}\left(a\left(x_{B}^{2}+z_{B}^{2}\right)+b z_{B}^{2}\right) \\
\dot{z}_{B} & =w_{B}+\varepsilon_{z}\left(z_{A}-z_{B}\right) \\
\dot{w}_{B} & =\mu z_{B}+z_{B}\left(a\left(x_{B}^{2}+z_{B}^{2}\right)+b x_{B}^{2}\right) .
\end{aligned}
$$

The coupling terms $\varepsilon_{x}\left(x_{B}-x_{A}\right)$ and $\varepsilon_{z}\left(z_{A}-z_{B}\right)$ can be interpreted as the feedback signals between both systems. These factors are equal to zero when complete synchronization is achieved and both systems reproduce the same trajectory on the synchronous manifold separately without any feedback between them. This kind of coupling extends the inner symmetries of each equations system to the coupled one which has a higher dimension. The results can be seen in Figure 2.
Variables $(x, y, z, w)$ in A are completely synchronized to $(x, y, z, w)$ in $\mathrm{B}$. This mean that $x_{A}$ is complete synchronized to $x_{B}$ and so on. Temporal signals displaying the synchronized state will be shown in the next paragraph together with effects of a driving signal.

Figure 2a displays the Fourier spectrum for one variable (variable $x_{A}$ ) and low frequencies detail is in Figure $2 \mathrm{~b}$. This figure shows a characteristic spectrum of a chaotic signal. Synchronization can be appreciated in Figure 2c, where the synchronization error function against time goes to zero after a short transient.

This information, (that can be easily obtained from an experiment), is not sufficient if we need to choose a frequency for driving the system. As in many other cases (like in the Rössler attractor for the "funnel" parameters), here also is not possible to construct a Poincaré section, and under these restrictions we cannot define an "analytical phase" [1].

To overcome this problem we constructed an histogram considering the period between two neighbors maximum (or minimum) obtained from a very long data file of the output signal. The amplitude in the histogram represent at each time value, the frequency (number of times) that this period value appears in the output signal, being the highest peak the most visited period. Resolution in time can be controlled choosing the width of the time interval to count, and in amplitude, it is one count over the total number of counts. A typical histogram constructed from the output signal of the coupled system is shown in Figure 2d. From this histogram, with periods distributed around two main peaks, we can obtain the most recurrent values of the frequencies appearing in the system.

\section{Driving with noiseless harmonic signals}

By using the information obtained from the recurrence time distribution is possible to analyze the effects of a noiseless harmonic signal injected to the synchronized system $\left(f_{E}\right.$ as shown in Fig. 1).

We will consider firstly the effects of signals with frequencies fitting one of the peaks observed in the time recurrence plots, to be compared with the effects obtained from the injection of other signal with a frequency in the middle of this peaks. In all the cases the amplitude of the signal injected will be the same, and a small fraction (1\%) of the variable value.

\subsection{Effect of driving with a "resonant" frequency}

We use here the word "resonance" in a wide sense, indicating that the period of the injected harmonic signal is coincident with one of the peaks in the histogram. Considering the recurrence plot in the Figure 2 d, firstly we injected a signal coincident, with the low-time peak in the recurrence histogram (that is, at high frequencies region $f=\frac{1}{t_{r}}$. In the values of the system: $t_{r}=5.3$ and consequently $\left.f_{E}=0.189\right)$ as it is represented overimposed in Figure 3. The amplitude of the signal injected is $1 \%$ of 


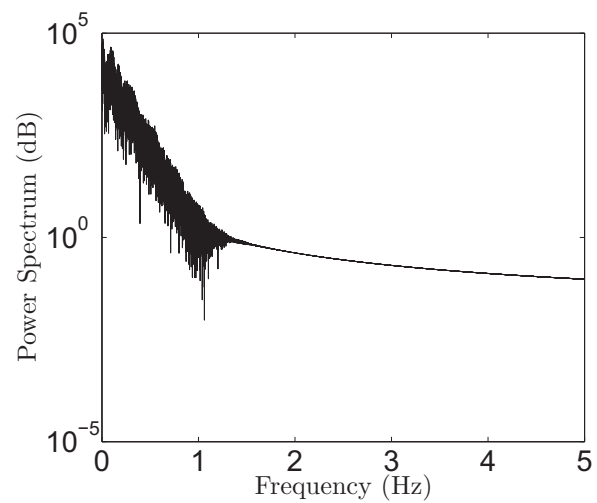

(a)

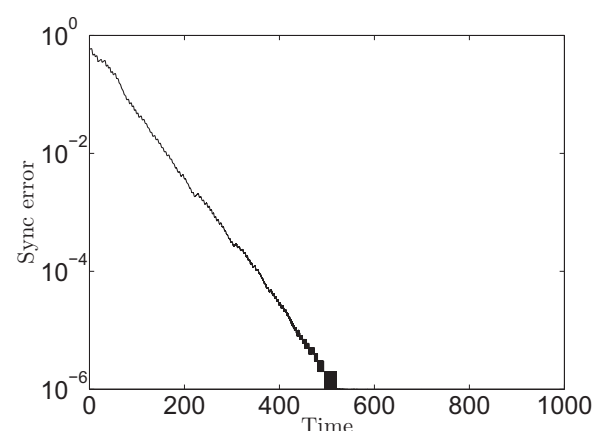

(c)

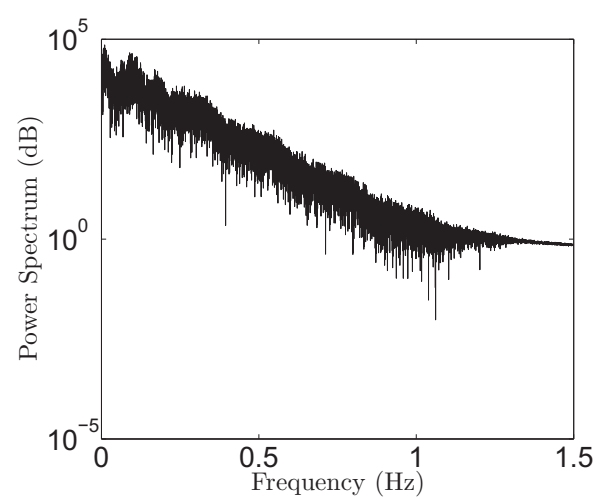

(b)

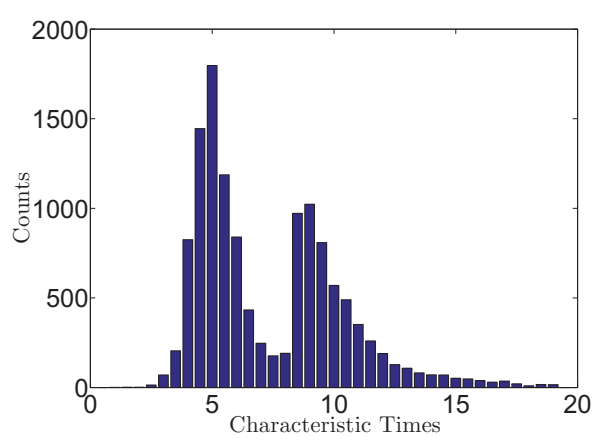

(d)

Fig. 2. (Color online) (a) Fourier spectrum for variable $x_{1}$. (b) Zoom on low frequencies. (c) Error function for synchronization in logarithmic scale. The function going to zero against time illustrate the necessary condition for CS. (d) Histogram of recurrent times (in seconds) that allow us to obtain the system characteristic times.

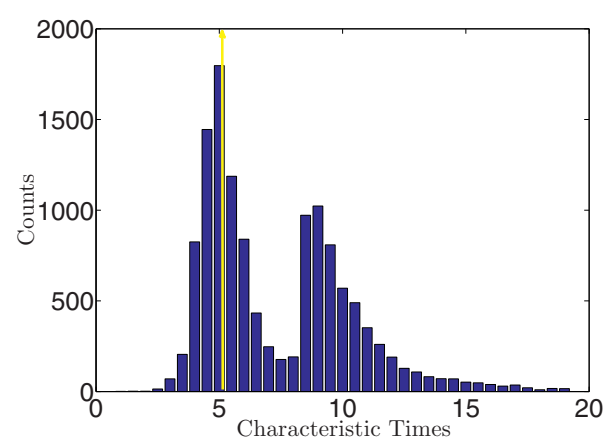

(a)

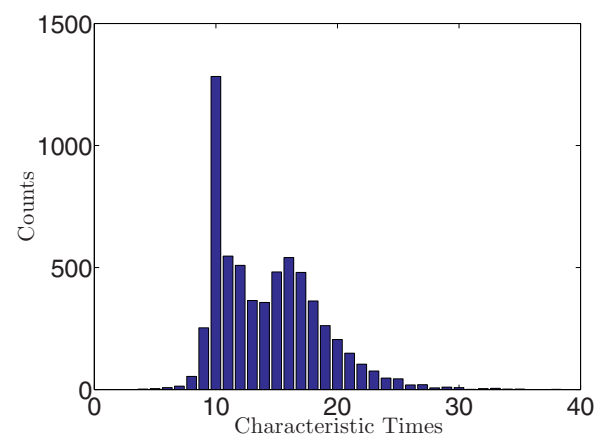

(b)

Fig. 3. (Color online) (a) Original histogram and harmonic signal $t_{r}=5.3\left(f_{E}=0.189\right)$. (b) New histogram after signal injection (note the change of scales).

the maximum value of variable $x$. This figure display in (b) the new histogram obtained after the injection of the signal. In Figure 4 appear in (a) the original Fourier spectrum of the signal $x$, and in (b) the spectrum after the injection of the signal. It can be seen in the figures that even being the injected amplitude a small fraction of the variable, it moves all the spectrum to the lower frequencies region, but this effect is less noticeable in the Fourier spectrum that in the histogram.

The results in the figures has been calculated for $x$ variable, but they are representative also for $y, z, w$.
In Figure 5 long sequences for all the variables are displayed beginning in (a) for the variable $x\left(x_{A}\right.$ in red, $x_{B}$ in blue). It should be noted that synchronization is kept even when the driving signal modify the spectrum profile.

Then, we injected a signal with frequency coincident with the peak in the long-times region of the histogram. In this case: $t_{r}=8.92$ and $f_{E}=0.112$, as it is represented in Figure 6a. The amplitude of the signal is the same that in the former case: $1 \%$ of the variable $x$. Figure $6 \mathrm{~b}$ shows the new histogram obtained. As before, in spite of the small amplitude, the driving signal moves the 


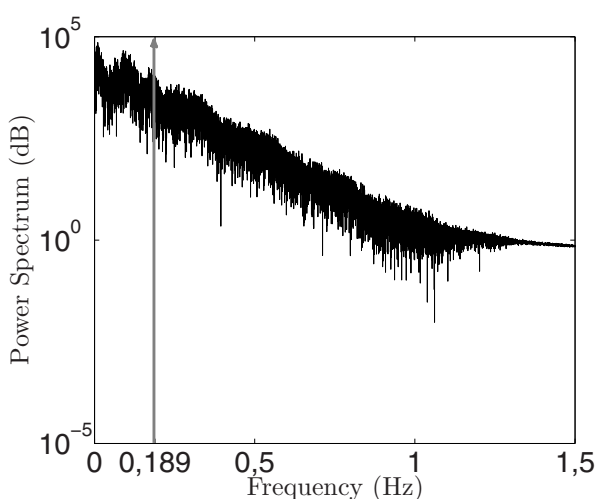

(a)

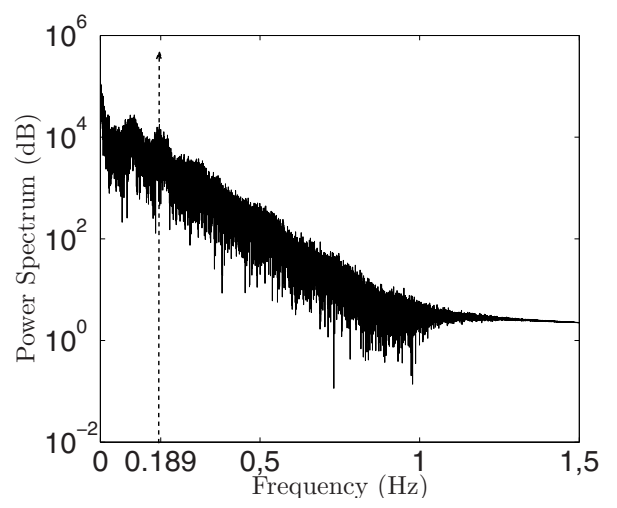

(b)

Fig. 4. (a) Original spectrum and harmonic signal $f_{E}=0.189\left(t_{r}=5.3\right)$. (b) New spectrum after signal injection.

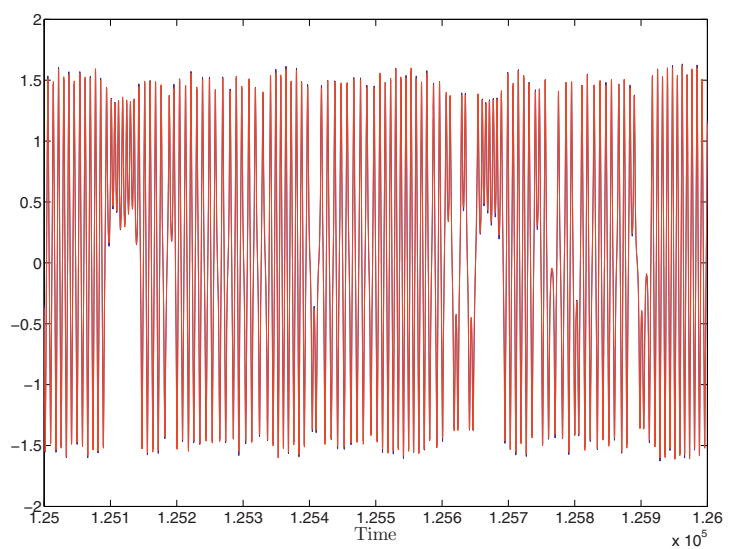

(a)

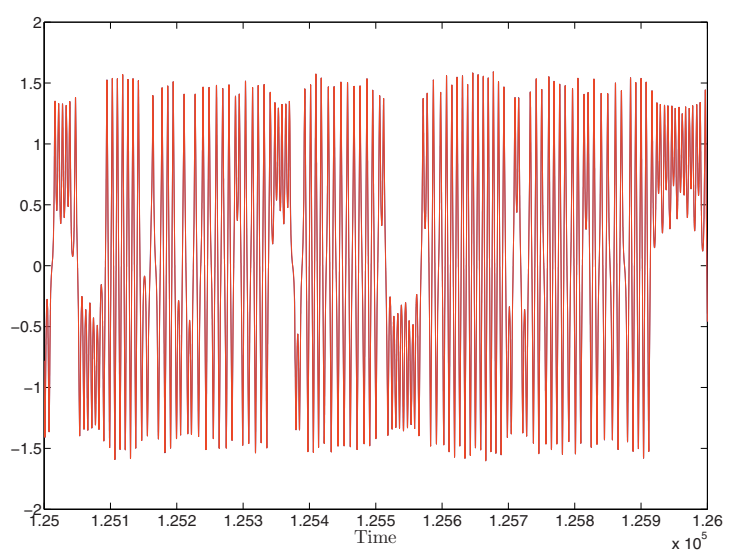

(c)

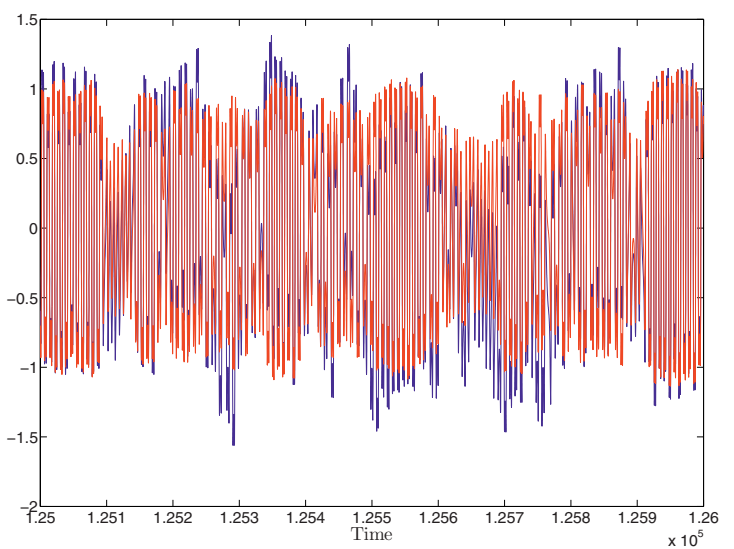

(b)

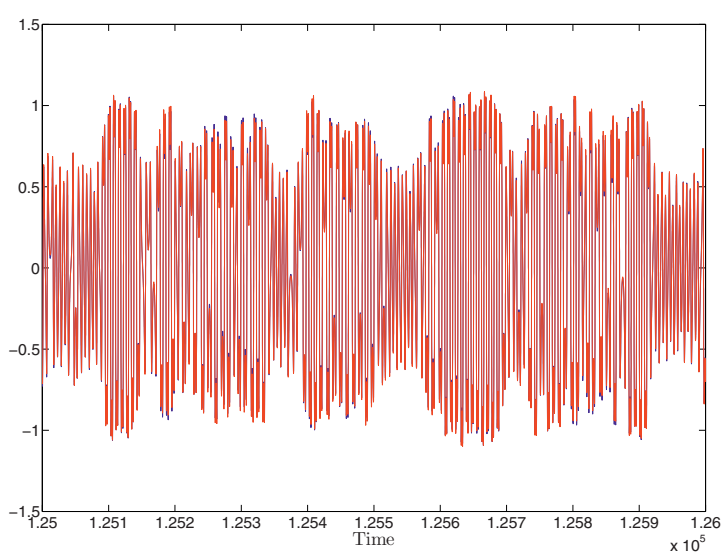

(d)

Fig. 5. (Color online) Temporal sequences for $x, y, z, w$ respectively for $f_{E}=0.189$ (system A in red, and B in blue).

center of the spectrum to the frequency region where this signal is injected. Efficiency is higher when the frequency is chosen at the peak of the histogram. As in the former case, histogram is moved to long times (low frequencies). In both cases, the oscillators tend to lock with the external frequency and complete synchronization is kept for variables $x$ and $z$. Variables $y$ and $w$ remain in phase synchronization as it is shown in Figure 7.

\subsection{Driving with a frequency in the middle of the time-distribution}

When a perturbation harmonic signal with frequency corresponding to the average in between the two peaks in the original histogram is injected, as it is shown in Figure 8a $\left(t_{r}=7.29\right.$, i.e., $\left.f_{E}=0.137\right)$ the effects are very noticeable. In (b) can be observed as the two peak structure of the 


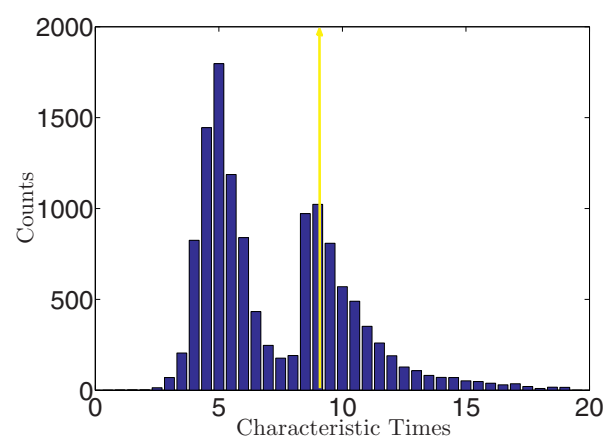

(a)

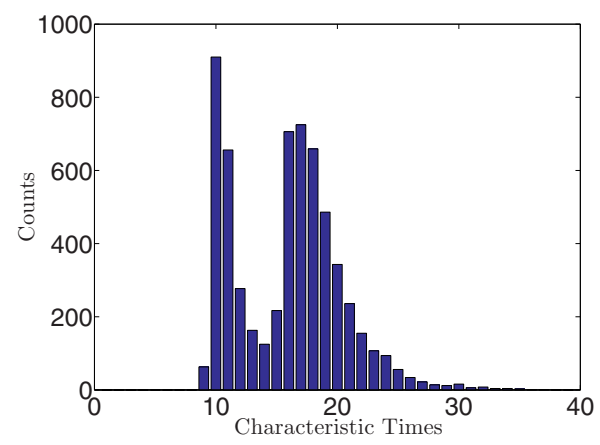

(b)

Fig. 6. (Color online) (a) Position of the injected signal in the original histogram $t_{r}=5.3 \mathrm{~s}\left(f_{E}=0.112\right)$. (b) Histogram after the injection of the signal (note the change in scales).

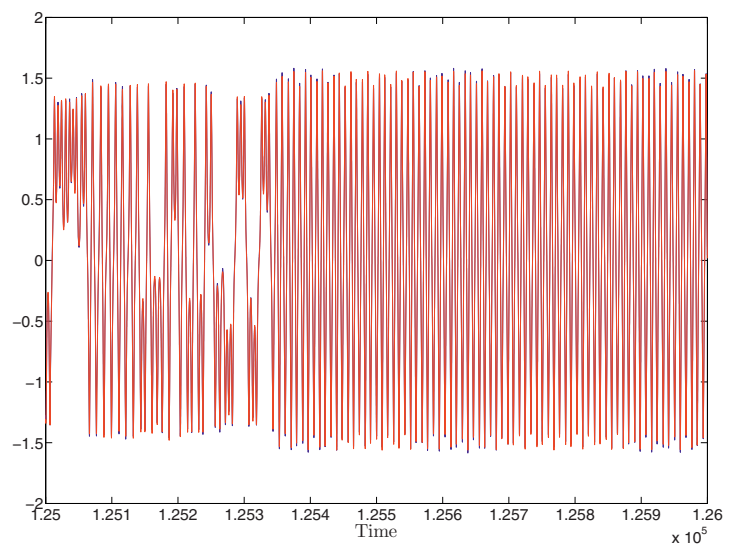

(a)

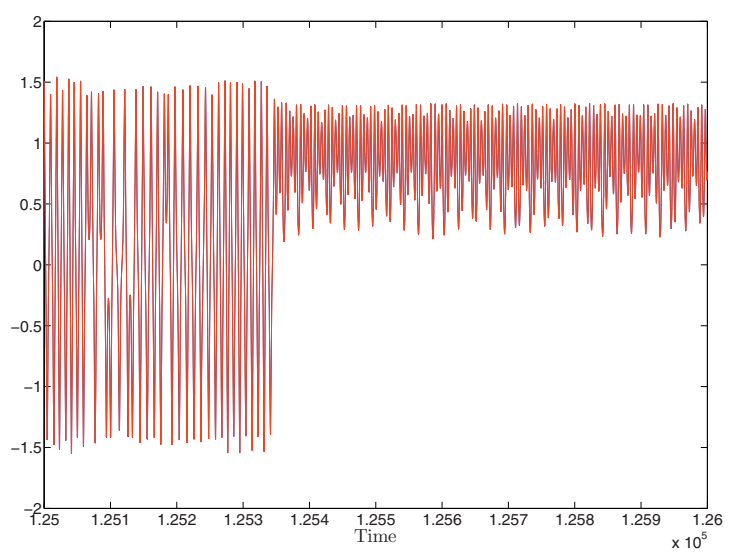

(c)

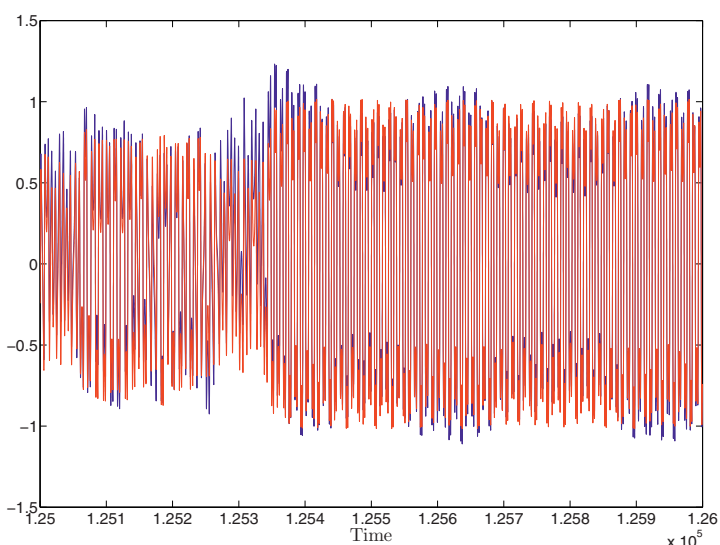

(b)

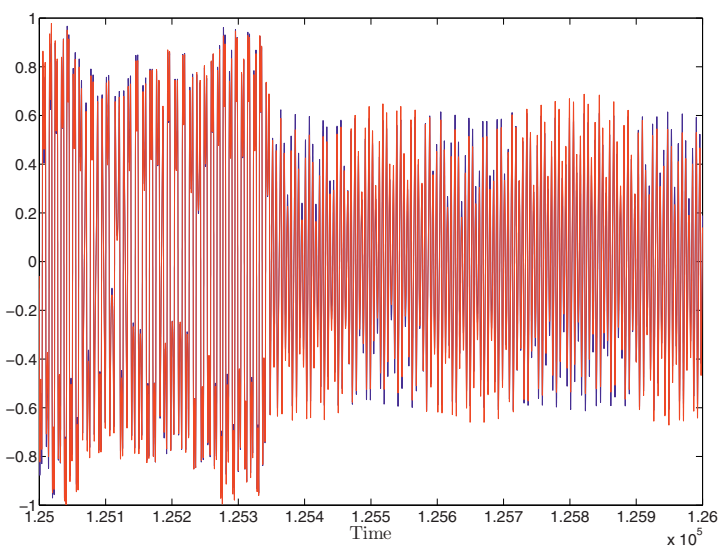

(d)

Fig. 7. (Color online) Temporal sequences for $x, y, z, w$ respectively for $f_{E}=0.112$ (system A in red, and B in blue).

initial time-recurrence distribution is kept, but compacted in a narrower band and moved to higher frequencies.

The results of the signal injected are shown in Figure 9. In (a) we display the original Fourier spectrum for the variable $x$ in the coupled system and the position of the injected signal. In (b) appears the new Fourier spectrum, obtained after the perturbation. There are strong differences with the unperturbed system (sharper frequen- cies and harmonics appears that appear overimposed to a wide band spectrum). This indicate that a weak signal has produced a new dynamic state. The corresponding time sequences of the variables are shown in Figure 11, note that complete synchronization is preserved for all the variables.

The transition to the new dynamical state can be seen in the plane $y$ vs. $x$ of the phase space diagram appearing 


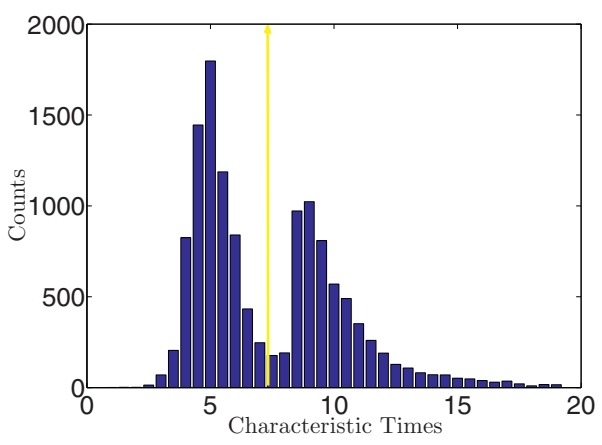

(a)

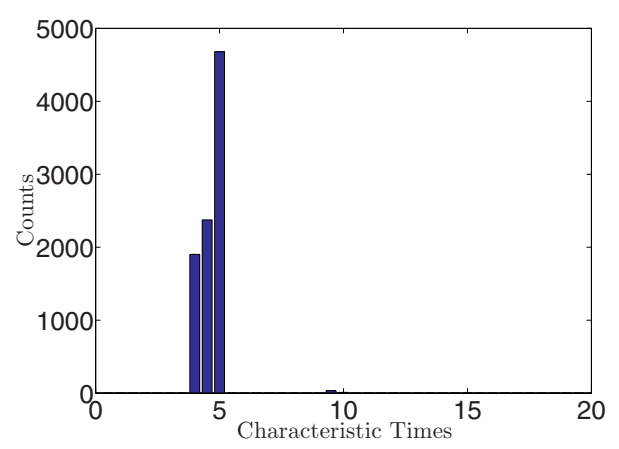

(b)

Fig. 8. (Color online) (a) Position of the injected signal in the original histogram $t_{r}=7.29\left(f_{E}=0.137\right)$. (b) Histogram after the injection of the driving signal (note the change in vertical scale).

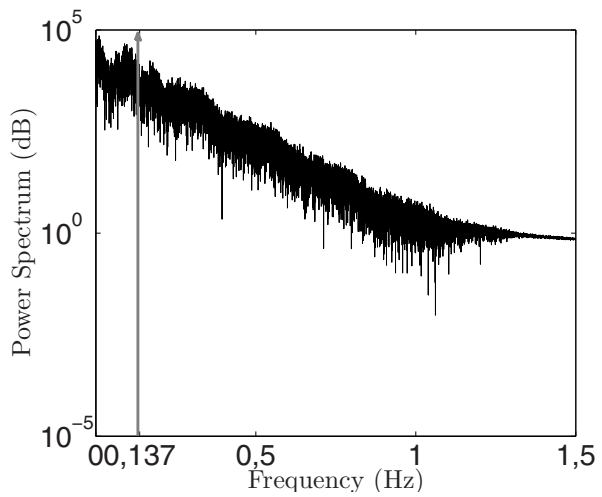

(a)

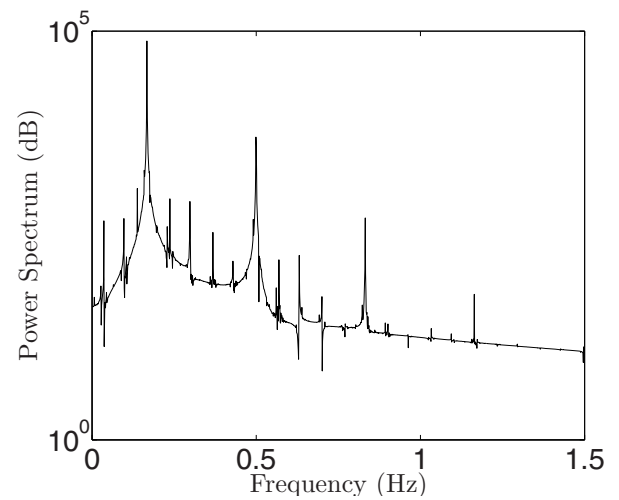

(b)

Fig. 9. (a) Original Fourier spectrum indicating the position of the frequency of the signal injected $f_{E}=0.137\left(t_{r}=7.29\right)$. (b) Spectrum obtained after the injection of the perturbation signal with (amplitude 1\% of the maximum value in $x_{A}$ ).

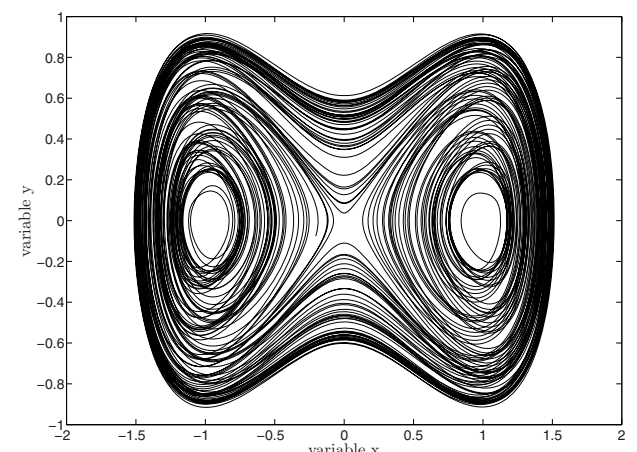

(a)

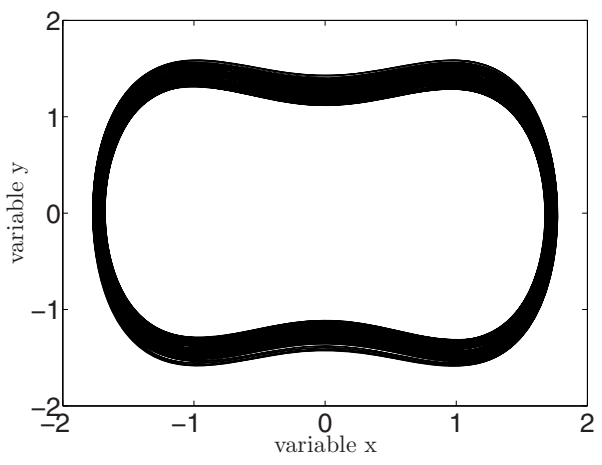

(b)

Fig. 10. (a) Original phase space $x$ vs. $y$. (b) Phase space transformation after the injection signal at $f_{E}=0.137$. All the variables remain completely synchronized.

in Figure 10. This plane is representative of the space phase (that is four dimensional [22]). The new narrow band Fourier spectrum and the diagram correspond to a more symmetrical and less complex dynamics with the signal remaining in the heteroclinic connection between the two asymmetrical attractors.

The change in dynamics of chaotic attractors by injection of weak harmonic signals have been studied pre- viously, i.e. $[23,24]$, appearing new states, including chaos suppression, bifurcations, intermittences, etc., as it was mentioned. A very clear analysis of the effects of signal injection to low dimensional systems (Lorentz, Rössler, Van der Pol and Takens Bogdanov) related with symmetries, can be found in [25] for a single attractor. Injection in coupled systems (two couped Van der Pol) [15] also display different dynamical states in a phase diagram (in 


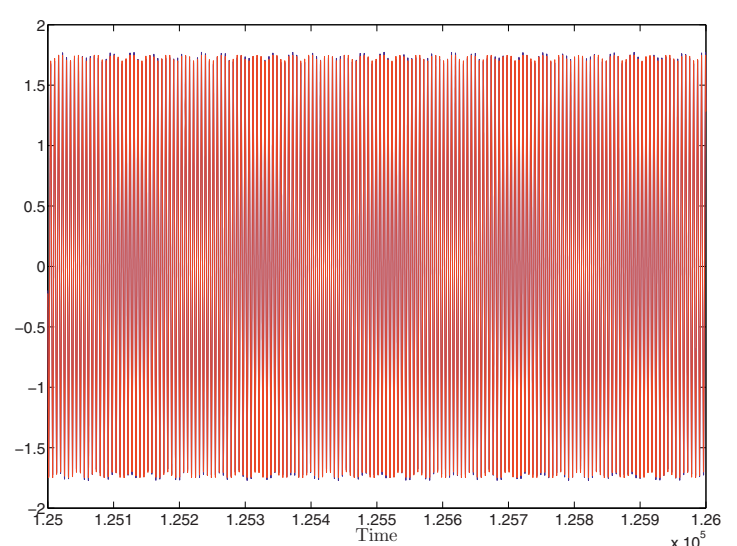

(a)

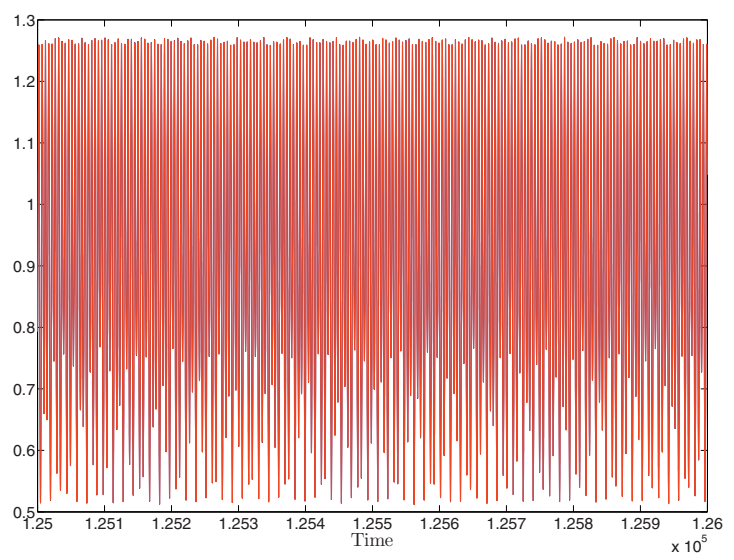

(c)

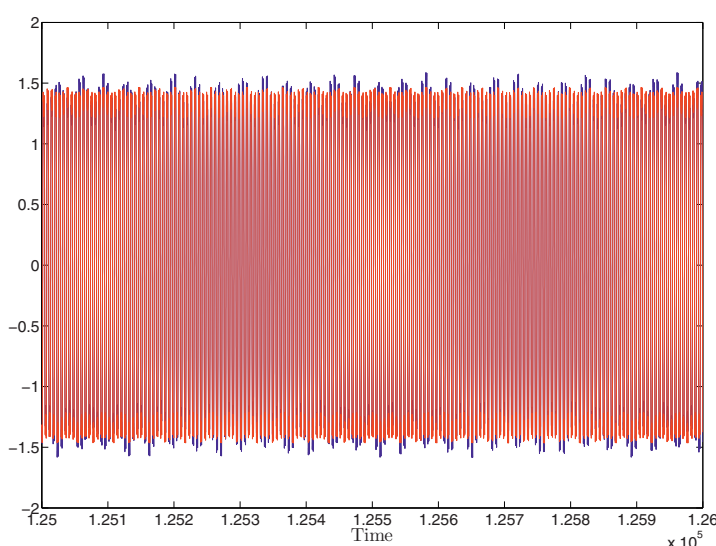

(b)

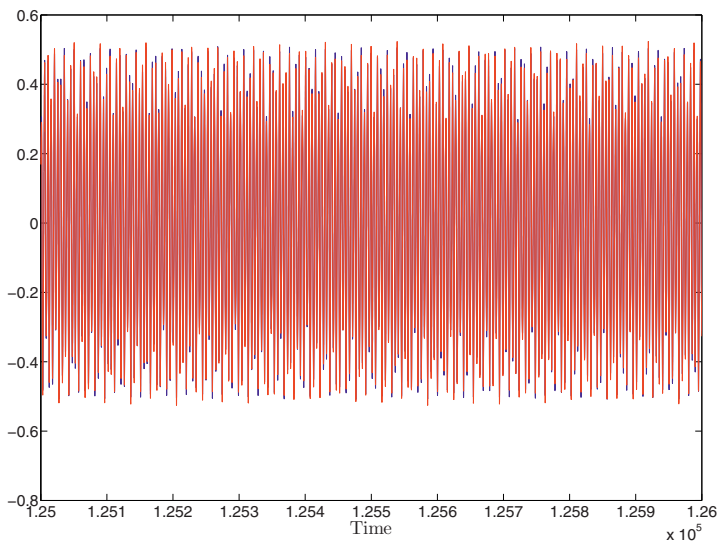

(d)

Fig. 11. (Color online) Temporal sequence for $x, y, z, w$ respectively for $f_{E}=0.137$.

the parameter space). The hyperchaotic system presented here is the coupling of two previously chaotic systems in a complete synchronization state. This complex system, as the previous cited, also display changes in the dynamic state by the injection of a weak harmonic signal and in a certain sense, this paper can be considered an extension of these previous works to an hyperchaotic high dimensional system. It should be remarked here, that the injection of a small signal in this case, even changing the dynamical state of the coupled chaotic systems, the transition to the new attractor is without chaos suppression, and with all the variables of the original two chaotic systems remaining in complete synchronization (Fig. 11).

\section{Noise effects}

We consider also the effect of a noisy signal in the last example (frequency of the injected signal centered in the time-recurrence distribution) and considering two different kinds of harmonic signals with added noise. Firstly, we add a uniform amplitude noise (10\% of the injected signal), covering uniformly all the frequency band centered around the frequency of the forcing signal, and then, a signal $x$ coming from a Rössler attractor centered in the same frequency with a maximum amplitude less than $10 \%$ of the driving signal amplitude (that is, in both cases the amplitude added is 0.001 of the variable $x$ ).

\subsection{Uniform noise}

The effects of adding uniform noise centered around the frequency of the signal injected in $x\left(f_{E}=0.137\right)$ is shown in Figure 12. In (a) the histogram shows that a signal with noise partially destroys the effects obtained by injection signal observed with a noise-free signal. In (b) we observe that variables $x$ in A (red) and B (blue) systems remain in complete synchronization in spite of the noise presence.

It was verified that for lower values of noise in the signal, the dynamics shown in Figure 11 was recovered. It was checked to prevent numerical artifacts.

\subsection{Injection signal from a Rössler attractor}

Using the output $x$ obtained from a Rössler attractor to add as a complex signal to the injection signal $f_{E}$ with an amplitude similar to the noise amplitude (10\%). As in the former case, we want to observe if there are some 


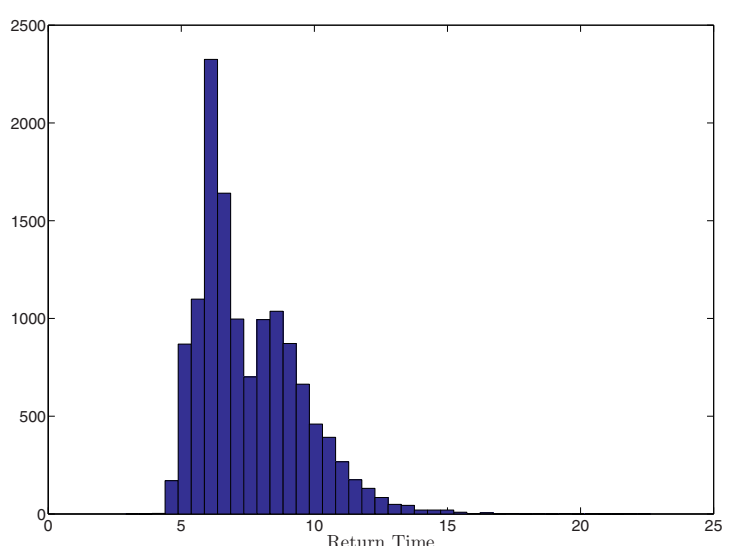

(a)

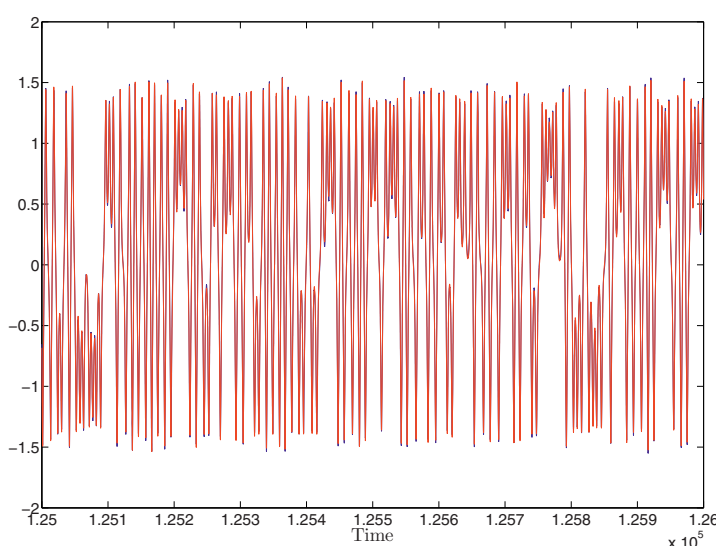

(b)

Fig. 12. (Color online) (a) Histogram for an injected signal at $f_{E}=0.137$ with $10 \%$ in amplitude of noise added to $f_{E}$. (b) Variables $x_{A}=x_{B}$ complete synchronized.

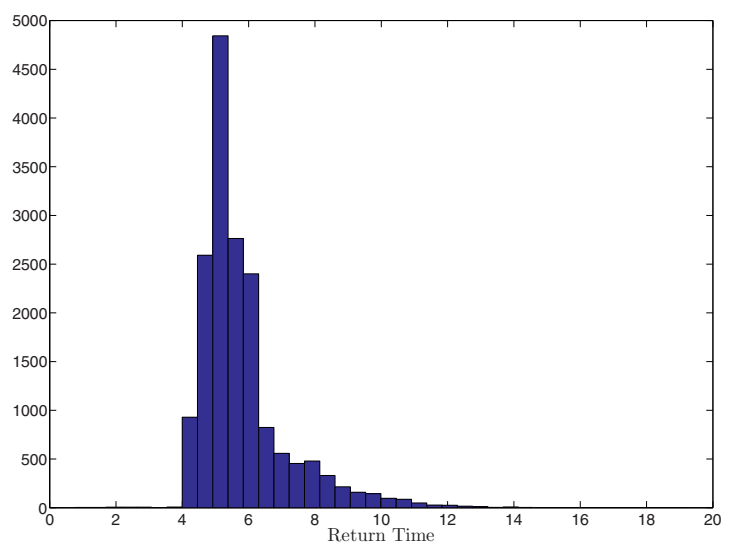

(a)

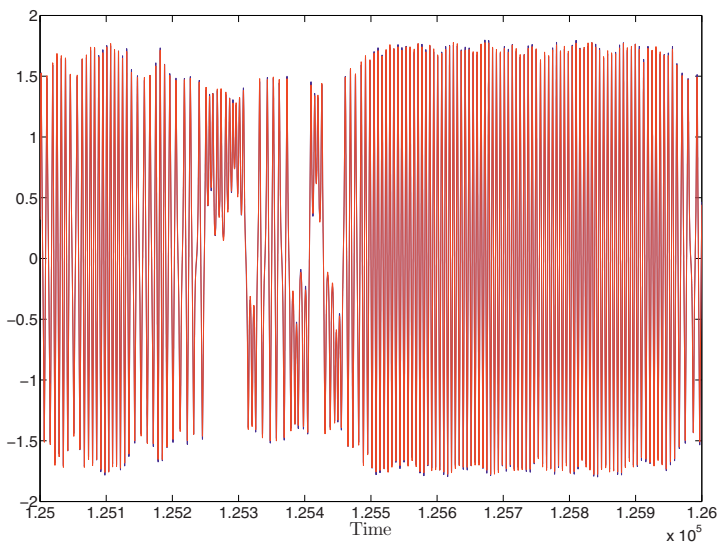

(b)

Fig. 13. (Color online) (a) Histogram for a signal injected at $f_{E}=0.137$ with correlated noise. (b) Time sequences for variables $x_{A}=x_{B}$.

difference in the effects between a small chaotic (deterministic) signal, and a noise without correlation. Amplitude used in both cases are similar, and the results obtained are shown in Figure 13. In (a) we observe that the effect of the injection signal with the chosen frequency $\left(f_{E}=0.137\right)$ does not disappear completely with addition of this kind of noise. This is a different behavior from the former case (noise uncorrelated). New intermittency regions interrupting the dynamics in the heteroclinic connection appear as the main effect (see Fig. 13b compared to Fig. 11a), but keeping the complete synchronization state, an intermediate dynamics between noise-free signal and a driving signal with uniform noise added.

\section{Conclusions}

We presented here the effects of a harmonic signal injection in a hyperchaotic dynamic system composed by two previously synchronized Takens-Bogdanov systems. Dynamics was set in complete synchronization regime and in the heteroclinic connection condition by adjusting parameters. This system was used because it has been demonstrated to be a useful model in some thermoconvective patterns appearing in experiments with square symmetry imposed by the lateral boundary conditions. To control this system with an external signal it is necessary to have some criteria to select the injection frequency. This information cannot be obtained clearly from the Fourier spectrum neither in numerical systems nor in experiments. Another problem in this system, as in others like the well known Rössler attractor in the "funnel" parameters conditions, is that topology in this higher dimension system inhibits to construct Poincaré sections to study the phase.

To select adequately the frequency for the injection signal and to avoid trial and error procedures, we proposed here to take profit from histograms constructed ad-hoc. The amplitude in this histograms represents the number of times that appear a period interval between two neighbours maximum (or minimum), when are measured from a long temporal sequence obtained in the numerical model or from a variable output in experiments. It represents the 
distribution of the most characteristics times appearing in the sequence. By using this information, we analyzed the effects of injection of harmonic signals with different frequencies on the system, coincident or not with the characteristic times. The results obtained and the addition of noise to the signals have been discussed. Different states in the chaotic dynamics are observed and, in spite of the hyperchaotic character of this system, we have shown that some control can be obtained with very small signal amplitude $(1 \%)$, preserving the complete synchronization state for all the variables of the system.

H. Mancini is indebted to Prof. P. Coullet for his many interesting insights in the physics of non-linear chaotic systems. He also kindly thanks the organizers of the "XII International Workshop on Instabilities and Nonequilibrium Structures" and all scientific colleagues from Chile, who go on with their efforts in spite of the terrible earthquakes suffered this year.

We also thank Wenceslao González-Viñas for many helpful discussions, and MEC Project FIS2008-01126 Spain and a PIUNA Project, University of Navarra, for financial support.

Gerard Vidal has been supported by a grant from "Asociación de Amigos", University of Navarra.

\section{References}

1. M.G. Rosenblum, A.S. Pikovsky, J. Kurths, Phys. Rev. Lett. 78, 4193 (1997)

2. D. Maza, A. Vallone, H.L. Mancini, S. Boccaletti, Phys. Rev. Lett. 85, 5567 (2000)

3. E.L. Koshmieder, Bénard Cells and Taylor Vortices (Cambridge University Press, 1993)

4. M.C. Cross, P.C. Hohenberg, Rev. Mod. Phys. 65, 851 (1993)

5. S. Boccaletti, C. Grebogi, Y.-C. Lai, H. Mancini, D. Maza, Phys. Rep. 329, 103 (2000)
6. A. Bernardini, J. Bragard, H. Mancini, Math. Biosci. Eng. 1, 339 (2004)

7. E. Schöll, H.G. Schuster, The Handbook of Chaos Control (Wiley-VCH, 2007)

8. R.O. Gregoriev, Physica D 140, 171 (2000)

9. A. Handel, R.O. Gregoriev, Phys. Rev. E 72, 066208 (2005)

10. R.O. Gregoriev, M.C. Cross, H.G. Schuster, Phys. Rev. Lett. 79, 2795 (1997)

11. S. Boccaletti, V. Latora, Y. Moreno, M. Chavez, D.-U. Huang, Phys. Rep. 424, 175 (2006)

12. T. Ondarçuhu, G.B. Mindlin, H.L. Mancini, C. PérezGarcía, Phys. Rev. Lett. 70, 3892 (1993)

13. T. Ondarçuhu, G.B. Mindlin, H.L. Mancini, C. PérezGarcía, J. Phys. Cond. Mat. 6, A427 (1994)

14. M. Huerta, D. Krmpotic, G.B. Mindlin, H. Mancini, D. Maza, C. Pérez-García, Physica D 96, 200 (1996)

15. V. Anishenko, S. Asthakov, T. Vadivasova, Europhys. Lett. 86, 30003 (2009)

16. G.B. Mindlin, Ondarcuhu, H. Mancini, C.P. Garcia, A. Garcimartin, Int. J. Bifurc. Chaos 4, 1121 (1994)

17. O. Rössler, Phys. Lett. A 71, 155 (1979)

18. G. Vidal, H. Mancini, Int. J. Bifurc. Chaos 19, 719 (2009)

19. H.G. Schuster, An introduction to Deterministic Chaos (Wiley-VCH, 2005)

20. J. Milnor, Commun. Math. Phys. 99, 177 (1985)

21. J. Bragard, G. Vidal, H. Mancini, C. Mendoza, S. Boccaletti, Chaos 17 (2007)

22. G. Vidal, H. Mancini, Int. J. Bifurc. Chaos 20, 885 (2010)

23. M.S. Baptista, I. Caldas, Nonlinear Dyn. 17, 119 (1998)

24. R. Chacon, Phys. Rev. Lett. 86, 1737 (2001)

25. R. Gilmore, C. Letellier, The symmetry of Chaos (Oxford Univ. Press, 2007)

26. D. Maza, B. Echebarría, C. Pérez-García, H.L. Mancini, Phys. Scr. T67, 82 (1996)

27. S. Boccaletti, The synchronized dynamics of complex systems, Monograph Series on Nonlinear Sciences and Complexity (Elsevier, 2008), Vol. 6

28. H. Mancini, D. Maza, Phys. Rev. E 55, 2757 (2000) 